\title{
Monocular Vision-based Aircraft Ground Obstacle Classification*
}

\author{
Péter Bauer ${ }^{1}$, Bálint Vanek ${ }^{1}$ and József Bokor ${ }^{2}$
}

\begin{abstract}
This article presents the first steps towards extending the applicability of the author's monocular vision-based aircraft sense and avoid method for steady ground obstacles. The goal is to decide if a ground obstacle is a collision threat or not. The focus of the development is real-time onboard applicability that's why simple calculations are proposed. After extending the calculation formulae for steady obstacles the results of a software-in-the-loop simulation campaign are presented for car and tower obstacles. The results are all acceptable so further developments will target a proper avoidance strategy and real flight tests.
\end{abstract}

\section{INTRODUCTION}

Sense and avoid (S\&A) capability is a crucial ability for the future unmanned aerial vehicles (UAVs). It is vital to integrate civilian and governmental UAVs into the common airspace according to [1] for example. Usually S\&A is understood as the sensing and avoidance of aerial vehicles, however in case of low level flight with small UAVs the avoidance of ground obstacles - such as transmission towers, tower-cranes, smokestacks or even tall tress - can be also vital to integrate them into the airspace.

This means that a small UAV's S\&A system should be prepared also to detect and avoid ground obstacles. Considering the size, weight and power constraints a monocular visionbased solution can be cost and weight effective therefore especially good for small UAVs [2], [3], [4].

Placing a vision system onboard can also help the obstacle avoidance and landing of manned aircraft as the EU-Japan H2020 research project [5] shows through its research goals. During landing the presence of ground vehicles on the runway can also be dangerous and should lead to a goaround.

In the literature, the detection and avoidance of ground obstacles is discussed for example in [6], [7], [8], [9] and [10].

[6] Proposes a stereo vision-based obstacle avoidance scheme for ground vehicles. [7] discusses path planning to avoid obstacles with known position. [8] discusses monocular SLAM-based obstacle avoidance applying also an altitude sonar. Real flight test results are also presented with a

\footnotetext{
*The research leading to these results has received funding from the European Union's Horizon 2020 research and innovation programme under grant agreement No. 690811 and the Japan New Energy and Industrial Technology Development Organization under grant agreement No. 062600 as a part of the EU/Japan joint research project entitled 'Validation of Integrated Safety-enchanced Intelligent flight cONtrol (VISION)'.

${ }^{1}$ Péter Bauer and Bálint Vanek are with Institute for Computer Science and Control, Hungarian Academy of Sciences (MTA SZTAKI), Budapest, Hungary peter.baueresztaki.mta.hu and vanek.balintesztaki.mta.hu

${ }^{2}$ József Bokor is with MTA-BME Control Engineering Research Group bokoresztaki.mta.hu
}

quadrotor helicopter and applying ground station-based calculations. [9] proposes a laser range finder-based method to detect and avoid static and dynamic obstacles with a UAV using a reactive path planner. Real flight test results are presented. [10] proposes an obstacle range estimation solution based-on aircraft velocity and the numerical differentiation of yaw angle and obstacle bearing angle. This solution can give uncertain results because of the numerical differentiation in case of noisy measurments. Another restriction is that a close to zero pitch angle is assumed. Then the article proposes a control solution to avoid multiple obstacles and presents real flight test results.

Previous works of the authors of this article [11], [12], [13] focused on the S\&A of aerial vehicles applying monocular camera system with onboard image processing and restricting aircraft movement to constant velocity and straight trajectories. No other assumption was done and no numerical differentiation is required in the proposed solution which can be an advantage compared to [10]. That's why this article focuses on the modification of previous results considering steady obstacles to see how effective the derived solution can be in the classification of ground obstacles. Constant own velocity and straight trajectory are still assumed. In the simulation test campaign two obstacle categories are considered, tower-like objects and cars as these are the main contingencies. The structure of the paper is as follows.

Section II summarizes the previous developments of the authors and presents their extension for steady obstacles. Section III first presents the software-in-the-loop (SIL) simulation setup, then evaluates the results of collision possibility and parameter estimation in case of a car and a tower (cylinder). Finally, section IV concludes the paper.

\section{TTCPA AND CPA ESTIMATION IN CASE OF STEADY OBSTACLES}

The results of previous developments in [11], [12], [13] are summarized here and modified to consider steady obstacles. Fig. 1 shows the definition of time to closest point of approach (TTCPA) and closest point of approach (CPA) in case of a steady obstacle.

The parameters are defined relative to the trajectory coordinate system (defined later in II-A) of the own aircraft, TTCPA is the time when the obstacle crosses the trajectory $\mathrm{X}$ axis and $X_{a}$ is the absolute distance between aircraft and obstacle at this point. $R$ is the characteristic size of the obstacle.

Considering oblique camera placement relative to the trajectory system the projection model of the obstacle in the horizontal plane (assuming a disc cross section) is shown in Fig. 2 where $X_{C}, Z_{C}$ is the camera coordinate system. 


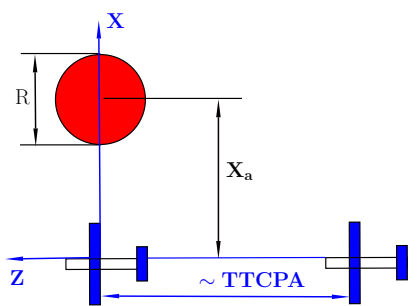

Fig. 1: Define TTCPA, $C P A=X_{a} / R$ (obstacle red circle, own aircraft blue from right)

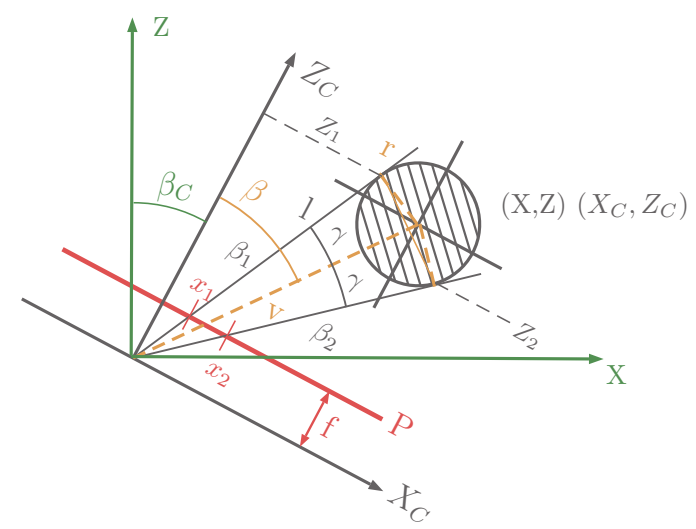

Fig. 2: Oblique camera disc projection model

The consideration of this disc projection model together with the $\beta_{C}$ camera angle and $V_{x}$ side and $V_{z}$ forward relative velocity components leads to the following formulae in [12].

$$
\begin{aligned}
& \bar{S}_{x}=S_{x}\left(\cos \beta_{1}+\cos \beta_{2}\right)=\frac{2 f R}{Z_{C}} \\
& \bar{x}=x\left(1-\frac{\bar{S}_{x}^{2}}{16 f^{2}}\right)=f \frac{X_{C}}{Z_{C}}
\end{aligned}
$$

Where $S_{x}$ and $x$ are the horizontal size and centroid position of the disc in the image, $\beta_{1}, \beta_{2}$ are the horizontal view angles of the edges of the disc image (see the figure), $f$ is camera focal length and $X_{C}, Z_{C}$ are the distances of the disc from the own aircraft in the camera coordinate system. They can be expressed by $V_{x}, V_{z}, X_{a}, t_{C P A}$ (the latter is TTCPA) as follows:

$$
\begin{aligned}
& X_{C}=X_{a} \cos \beta_{C}-\left(V_{x} \cos \beta_{C}-V_{z} \sin \beta_{C}\right) t_{C P A} \\
& Z_{C}=X_{a} \sin \beta_{C}-\left(V_{x} \sin \beta_{C}+V_{z} \cos \beta_{C}\right) t_{C P A}
\end{aligned}
$$

Substituting the expressions of $X_{C}$ and $Z_{C}$ into the reciprocal and ratio of the expressions for $\bar{x}$ and $\bar{S}_{x}$ in (1) and considering $C P A_{x}=\frac{X_{a}}{R}$ one gets:

$$
\begin{aligned}
& \frac{1}{\bar{S}_{x}}=\frac{C P A_{x}}{2} \frac{\sin \beta_{C}}{f}-\frac{V_{x} \sin \beta_{C}+V_{z} \cos \beta_{C}}{2 f R} t_{C P A} \\
& \overline{\bar{x}}=\frac{C P A_{x}}{2} \cos \beta_{C}-\frac{V_{x} \cos \beta_{C}-V_{z} \sin \beta_{C}}{2 R} t_{C P A}
\end{aligned}
$$

In this system of equations the unknowns are $C P A_{x}$ and $t_{C P A}$ and the time varying terms are $\bar{x}, \bar{S}_{x}, t_{C P A}$. The other terms such as $f, \beta_{C}, V_{x}, V_{z}$ and $R$ are all constant.
Considering this and $t_{C P A}=t_{C}-t$ one gets $(t$ is actual time, $t_{C}$ is the time when intruder is closest to own aircraft (it is constant)):

$$
\begin{aligned}
& \frac{1}{\overline{\bar{S}}_{x}}=\frac{\sin \beta_{C}}{f} \frac{C P A_{x}}{2}-a_{1} t_{C}+a_{1} t=c_{1}+a_{1} t \\
& \frac{\bar{x}}{\bar{S}_{x}}=\cos \beta_{C} \frac{C P A_{x}}{2}-a_{2} t_{C}+a_{2} t=c_{2}+a_{2} t
\end{aligned}
$$

Making a simple least squares optimal line fit to the measured $\frac{1}{S_{x}}, \frac{\bar{x}}{S_{x}}, t$ paremeters will give $a_{1}, a_{2}, c_{1}, c_{2}$ and the following system of equations.

$$
\left[\begin{array}{cc}
\frac{\sin \beta_{C}}{f} & -a_{1} \\
\cos \beta_{C} & -a_{2}
\end{array}\right]\left[\begin{array}{c}
\frac{C P A_{x}}{2} \\
t_{C}
\end{array}\right]=\left[\begin{array}{l}
c_{1} \\
c_{2}
\end{array}\right]
$$

$C P A_{x}$ and $t_{C}$ (and so $t_{C P A}$ ) can be easily obtained from this. Considering now a steady obstacle means that there will be no side velocity component $\left(V_{x}=0\right)$ and the forward component $V_{z}$ is known as the own ground relative velocity. This leads to simplified expressions in (3) but has no effect on (4) and (5).

However, knowing the value of $V_{z}$ makes it possible to estimate the $R$ absolute size of the obstacle considering $a_{1}, a_{2}$.

$$
\begin{aligned}
& a_{1}=\frac{V_{z} \cos \beta_{C}}{2 f R}, \quad a_{2}=\frac{-V_{z} \sin \beta_{C}}{2 R} \\
& R=\frac{V_{z}}{4}\left(\frac{\cos \beta_{C}}{a_{1} f}-\frac{\sin \beta_{C}}{a_{2}}\right)
\end{aligned}
$$

Knowing the size of the obstacle makes it possible to estimate the absolute side distance $X_{a}$ and considering $t_{C P A}$ and the forward velocity $V_{z}$ the forward absolute distance $Z_{a}$ can be also estimated.

$$
X_{a}=R \cdot C P A_{x} \quad Z_{a}=V_{z} \cdot t_{C P A}
$$

Considering the vertical situation in the S\&A of aircraft intruders the vertical image centroid position $y$ and size $S_{y}$ together with similar formulae as in the horizontal situation can be considered. However, for ground obstacles the vertical parameters can be different. Considering the possible avoidance strategies the aircraft can fly around the obstacle or ascend above it. From this point of view its better to estimate the altitude of the top point of obstacle relative to the own aircraft. The related image parameter is the top coordinate $y_{T}$ as shown in Fig.s 3, 4.

The figures show that considering the horizontal paremeters in case of a tower-like object the average $S_{x}$ width and the horizontal centroid can be determined together with the coordinate of the top point $y_{T}$. In case of a car-like object the full horizontal size gives $S_{x}$ and the other coordinates are also the $x$ centroid position and $y_{T}$. 


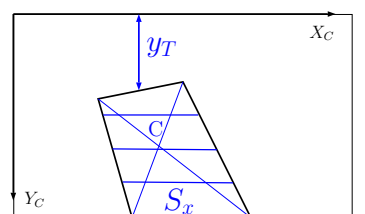

Fig. 3: Image parameters of a tower

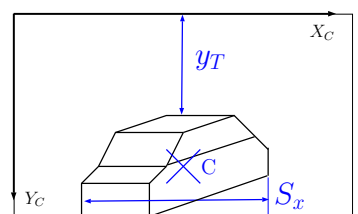

Fig. 4: Image parameters of a car
Considering the vertical pinhole camera projection formula one can determine the absolute $Y_{a}$ distance relative to the trajectory coordinate system if knows the forward distance $Z_{a}$ and the focal length of the camera.

$$
y_{T}=f \frac{Y_{a}}{Z_{a}}, \quad Y_{a}=\frac{y_{T} Z_{a}}{f}
$$

\section{A. Note about ego motion transformation}

In any S\&A task it is very important to calculate the position of the intruder/obstacle relative to a well defined coordinate system. As at this stage of developments straight aircraft trajectories are assumed it is straightforward to calculate every parameter relative to a trajectory coordinate system aligned with the own aircraft path. This will give CPA relative to the undisturbed path of the aircraft and so it can be decided if there is the need to leave the trajectory and make an avoidance maneuver. However, the aircraft rolls and pitches relative to the straight track and these motions will corrupt the images. To avoid this corruption ego motion compensation should be applied. Fig. 5 shows all of the coordinate systems considered in this procedure.

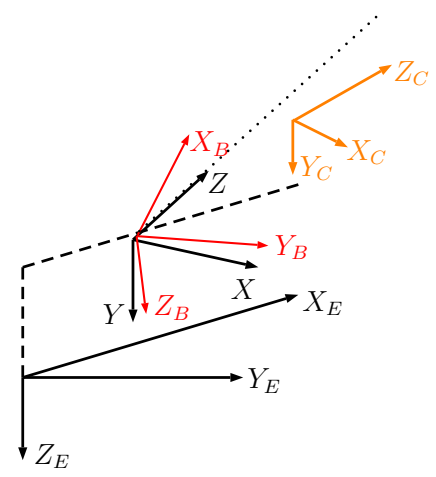

Fig. 5: The applied coordinate systems

$X_{E}, Y_{E}, Z_{E}$ is the Earth, $X, Y, Z$ is the trajectory ( $Z$ axis parallel with the straight trajectory (dotted line)), $X_{B}, Y_{B}, Z_{B}$ is the body and $X_{C}, Y_{C}, Z_{C}$ is the camera coordinate system. The goal is to transform the image coordinates to characterize the situation relative to the trajectory system as the image processing is done relative to this (see Fig. 2). The measured image point vector $P^{C}=\left[\begin{array}{lll}x & y_{T} & f\end{array}\right]$ should be first normalized as $\bar{P}^{C}=P^{C} /\left\|P^{C}\right\|_{2}$. Then it should be rotated with the $T_{B C}$ camera to body transformation matrix to get $\bar{P}^{B}$. In the next step rotation to the trajectory coordinate system should be done considering the Euler angles $(\phi, \theta, \psi)$ relating the body to the earth system and the horizontal direction of the trajectory system $\psi_{0}$ relative to the Earth. It is assumed that the trajectory is almost horizontal and so the trajectory system is only horizontally rotated relative to the earth. This way a body to trajectory transformation can be done considering $\phi, \theta, \psi-\psi_{0}$. This results in $\bar{P}^{T}$. This should be transformed back to the camera system with $T_{C B}$ to obtain image coordinates $Q$ relative to the trajectory system. The final step is the back scaling of this unit vector to have the $f$ focal length as its third coordinate: $\bar{Q}=\frac{Q}{Q(3)} f$. The image size coordinates are also transformed. The transformed coordinates are again denoted by $S_{x}, x$.

However, in case of a steady object's top vertical coordinate (7) applies the forward $Z_{a}$ distance along the $\mathrm{Z}$ axis of the trajectory system. This means that $y_{T}$ should be determined relative to the trajectory system instead of the camera as the camera can be further rotated (see Fig. 2) and thus give different $y$ image value. So in case of the vertical coordinate $\bar{P}^{T}$ should be directly scaled back $\left(P^{T}=\frac{\bar{P}^{T}}{P^{T}(3)} f\right)$ to get $y_{T}$.

\section{SIL SIMULATION RESULTS}

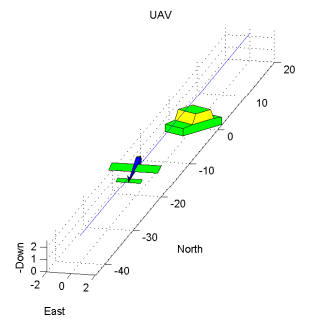

Fig. 6: SIL simulation of car approach

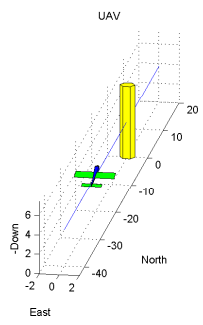

Fig. 7: SIL simulation of tower approach

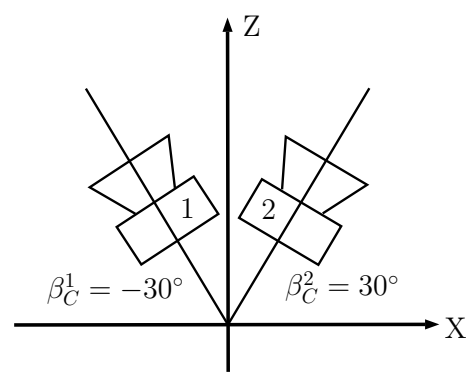

Fig. 8: The sketch of the onboard two camera system

A SIL simulation test campaign was done considering carlike (see Fig. 6) and tower-like (see Fig. 7) obstacles also and an own aircraft equipped with a two camera system (see Fig. 8). The horizontal field of view (FOV) of the cameras was $\pm 35^{\circ}$ while the vertical was $+45^{\circ}$ and $-25^{\circ}$ which means a downward looking camera. Pixelization errors were considered in the simulation of the camera system.

The aircraft was flown on a straight trajectory towards the obstacle applying autopilot for trajectory tracking. A Monte Carlo simulation was run for each (car / tower) case. The own velocity $\left(V_{o}\right)$ was $15 \mathrm{~m} / \mathrm{s}$ or $30 \mathrm{~m} / \mathrm{s}$. The glide slope was $0^{\circ}$, $3^{\circ}$ or $6^{\circ}$ satisfying the almost horizontal track assumption (no ascending was considered). The side distance from the 
obstacle was characterized by $C P A$ and the characteristic size. The considered $C P A$ values are $0,5,10$. In case of the car model the size was not changed the width of the car is $1.9 \mathrm{~m}$ which is also the characteritic size. In case of the tower the characteristic size is the diameter which is chosen from $1.5 \mathrm{~m}, 3 \mathrm{~m}, 6 \mathrm{~m}$. On the other hand the orientation of the car was chosen from $0^{\circ}, 30^{\circ}, 60^{\circ}, 90^{\circ}$ where $0^{\circ}$ means the nose towards the aircraft while $90^{\circ}$ means the side of the car towards the aircraft. The last parameter considered is the altitude where the aircraft is above the obstacle. This was considered as a given percentage of the height of the object. The height of the car is fixed to $1.5 \mathrm{~m}$ and the considered precentages are $0 \%, 100 \%, 200 \%, 400 \%$. The height of the tower is size. 5 and the considered precentages are $0 \%, 50 \%, 100 \%, 150 \%$. The difference of the car and tower precentages is because of the large difference of their absolute heights. The car is very small and so there is no point in flying $0.75 \mathrm{~m}$ and then $1.5 \mathrm{~m}$ above the ground with the aircraft, the two cases are almost the same. Note that the given altitude values are only the references, the tracking autopilot of the aircraft follows it with some transient and tracking error. A threshold of 3 second was set for the collision decision in each simulation so when the estimated TTCPA value gets below this a decision is done and all the estimated parameters are compared to the real ones (errors are calculated). The CPA threshold was selected to be 7 to decide about the CPA 0 and 5 cases as collision and about the CPA 10 cases as no collision. Note that only the decision was done, no avoidance maneuver was started to be able to calculate the real TTCPA and CPA values.

The test results are plotted in histograms for the different estimation errors in Fig. 9 to 14 for the car and in Fig. 15 to 20 for the tower. The first plot shows the real TTCPA value when the decision is done. The second shows the ratio of the estimated and the real CPA values (if the real values are close to zero this ratio is not calculated). The third shows the side distance estimation errors in percent (if the real values are close to zero this ratio is not calculated). The fourth shows the size estimation errors in percent. The fifth shows the forward distance estimation errors in percent. Finally, the sixth shows the vertical distance estimation errors in meters. This is not in percent because the real values are close to zero several times and so the error percentages get very large.

In case of the car 288 simulations were done. Regarding the TTCPA values (Fig. 9) most of the real values (when the estimate gets below 3 secs) is between 2.5 and 3.5 secs which means that the estimation error is about $\pm 0.5 \mathrm{~s}$ in most of the cases which is a really good results. The CPA ratios (Fig. 10) are below 1.2 in most of the cases. The minimum values are about 0.3 and 0.5 for $15 \mathrm{~m} / \mathrm{s}$ and $30 \mathrm{~m} / \mathrm{s}$ own velocities respectively. The figure shows that CPA is usually underestimated which gives conservative results. This is underlined by the fact that collision is decided even for $C P A=10$ in all cases. This means that the CPA threshold can be decreased to 6 (considering the maximum 1.2 overestimation) to avoid car in all $C P A=5$ cases and do not avoid it in $C P A=10$ cases. Considering the side distance errors (Fig. 11) they are between $-60 /+50 \%$ and -
$60 /+20 \%$ which are very large ranges however, the majority of the values is between $\pm 20 \%$ which can be acceptable. The object size estimation errors (Fig. 12) can be large for $15 \mathrm{~m} / \mathrm{s}$ own velocity and are below $20 \%$ for $30 \mathrm{~m} / \mathrm{s}$. This can be caused by the larger movements between two image frames in the latter case which makes the line fitting in (4) more accurate. The majority of the forward distance errors (Fig. 13 ) is between $\pm 20 \%$ which can be acceptable. The majority of the vertical distance estimation errors (Fig. 14) is between $\pm 2 m$ which is a really good result.

Summarizing the results, the TTCPA and CPA estimates can be well used together with the vertical distance estimate to decide about the need for avoidance and design a safe avoidance maneuver. The estimated object size, side and forward distance values can only be used as approximations showing the order of magnitudes of these parameters.

In case of the tower 216 simulations were done. Regarding the TTCPA values (Fig. 15) most of the real values (when the estimate gets below 3 secs) is between 2.5 and 3.5 secs which means that the estimation error is about $\pm 0.5 \mathrm{~s}$ in most of the cases which is a really good results. The CPA ratios (Fig. 16) are below 1.2 and above 0.8 in all of the cases. This means a much better estimation compared to the car cases. This is underlined by the good decisions as in this case non-collision is decided for all $C P A=10$ values. Considering the side distance errors (Fig. 17) they are between $-15 /+50 \%$ and $30 /+15 \%$ which are better ranges then for the car but still very large. However, the majority of the values is between $\pm 15 \%$ which can be acceptable. The object size estimation errors (Fig. 18) can be large for $15 \mathrm{~m} / \mathrm{s}$ own velocity and are below $20 \%$ for $30 \mathrm{~m} / \mathrm{s}$ similarly to the car cases. The majority of the forward distance errors (Fig. 19) is between $\pm 10 \%$ which is better then for the car and is acceptable. The majority of the vertical distance estimation errors (Fig. 20) is between $\pm 3 \mathrm{~m}$ which is a really good result, but there are outliers as large as $15 \mathrm{~m}$. Examining the data in details shows that this is caused by the improper tracking of altitude by the autopilot in some cases. There are transients which cause unneccessary pitching motion and this leads to an uncertain estimation of the vertical distance despite the ego motion compensation (which is not perfect of course). After the altitude stabilizes the vertical distance estimate converges well to the real value, but this is too late in these cases, the collision decision is done earlier.

Summarizing the results, the TTCPA and CPA estimates are better then for the car and can be well used to decide about the need for avoidance and design a safe avoidance maneuver. The estimated object size, side and forward distance values are better then for the car but can only be used as approximations showing the order of magnitudes of these parameters. The excessive errors in the vertical distance estimate are because of the pitching transient dynamics of the aircraft in tracking the trajectory. After the transients the results are acceptable so these parameters can also be used if the aircraft is in steady trajectory flight. 

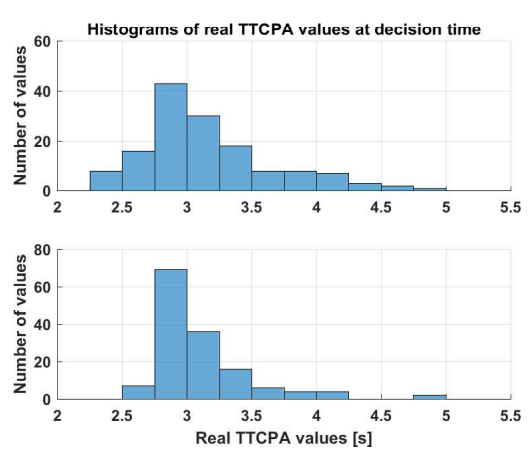

Fig. 9: Histogram of real TTCPA at decision for car (upper for $15 \mathrm{~m} / \mathrm{s}$ lower for $30 \mathrm{~m} / \mathrm{s} V_{o}$ )
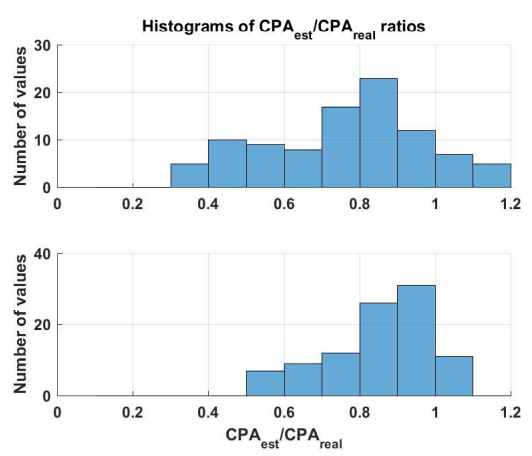

Fig. 10: Histogram of CPA ratios at decision for car (upper for $15 \mathrm{~m} / \mathrm{s}$ lower for $30 \mathrm{~m} / \mathrm{s} V_{o}$ )
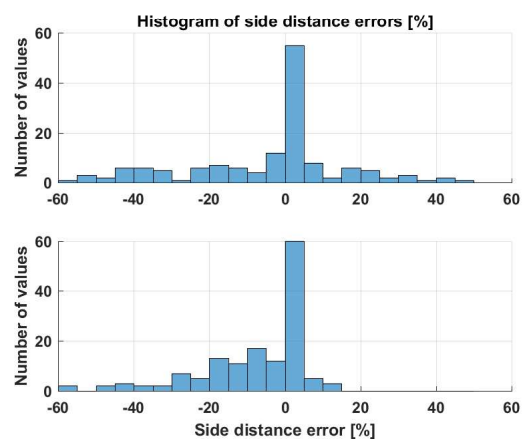

Fig. 11: Histogram of side distance estimation errors at decision for car (upper for $15 \mathrm{~m} / \mathrm{s}$ lower for $30 \mathrm{~m} / \mathrm{s} V_{o}$ )
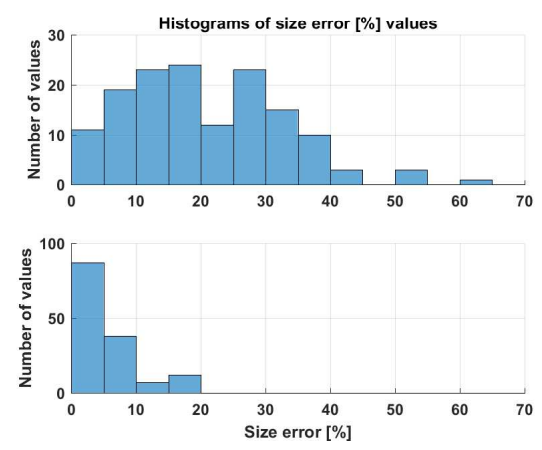

Fig. 12: Histogram of size estimation errors at decision for car (upper for $15 \mathrm{~m} / \mathrm{s}$ lower for $30 \mathrm{~m} / \mathrm{s} V_{o}$ )
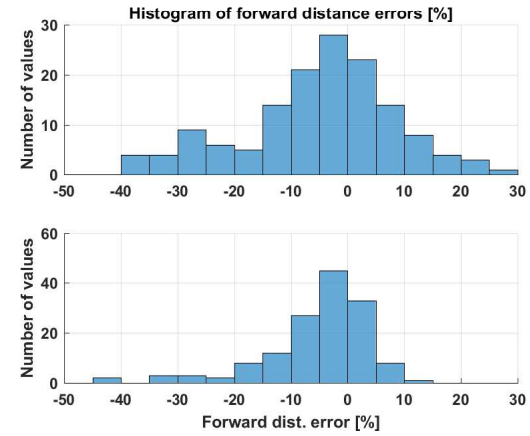

Fig. 13: Histogram of forward distance estimation errors at decision for car (upper for $15 \mathrm{~m} / \mathrm{s}$ lower for $30 \mathrm{~m} / \mathrm{s} V_{o}$ )
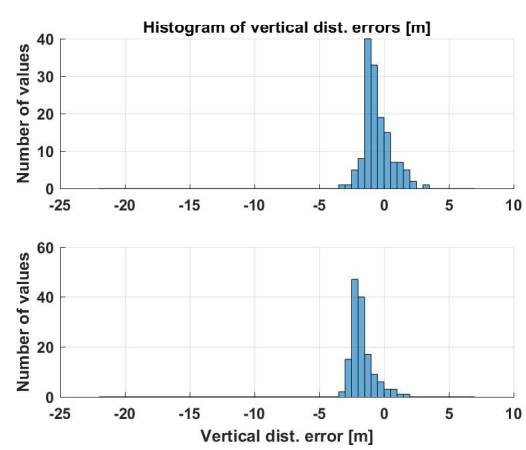

Fig. 14: Histogram of vertical distance estimation errors at decision for car (upper for $15 \mathrm{~m} / \mathrm{s}$ lower for $30 \mathrm{~m} / \mathrm{s} V_{o}$ )
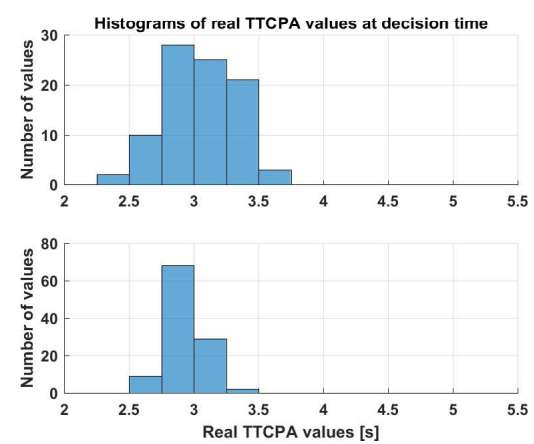

Fig. 15: Histogram of real TTCPA at decision for tower (upper for $15 \mathrm{~m} / \mathrm{s}$ lower for $30 \mathrm{~m} / \mathrm{s} V_{o}$ )
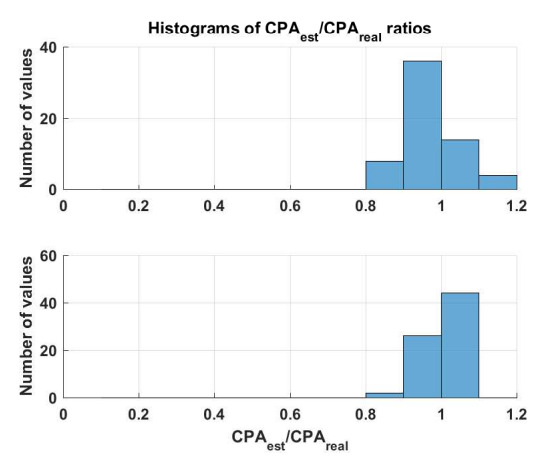

Fig. 16: Histogram of CPA ratios at decision for tower (upper for $15 \mathrm{~m} / \mathrm{s}$ lower for $30 \mathrm{~m} / \mathrm{s} V_{o}$ ) 

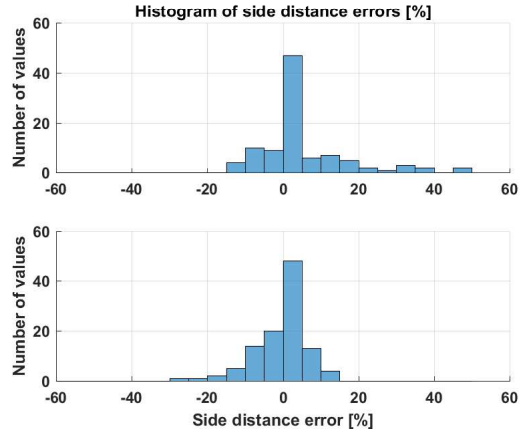

Fig. 17: Histogram of side distance estimation errors at decision for tower (upper for $15 \mathrm{~m} / \mathrm{s}$ lower for $30 \mathrm{~m} / \mathrm{s} V_{o}$ )
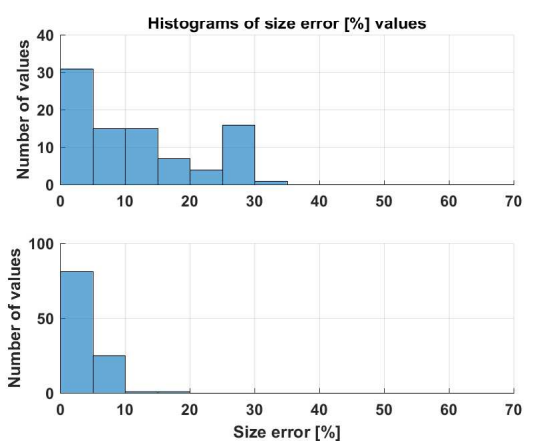

Fig. 18: Histogram of size estimation errors at decision for tower (upper for $15 \mathrm{~m} / \mathrm{s}$ lower for $30 \mathrm{~m} / \mathrm{s} V_{o}$ )
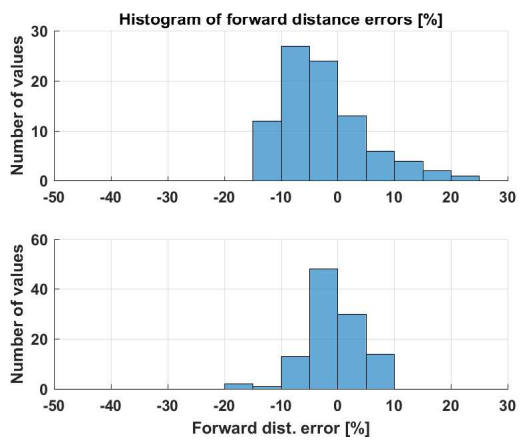

Fig. 19: Histogram of forward distance estimation errors at decision for tower (upper for $15 \mathrm{~m} / \mathrm{s}$ lower for $30 \mathrm{~m} / \mathrm{s} V_{o}$ )
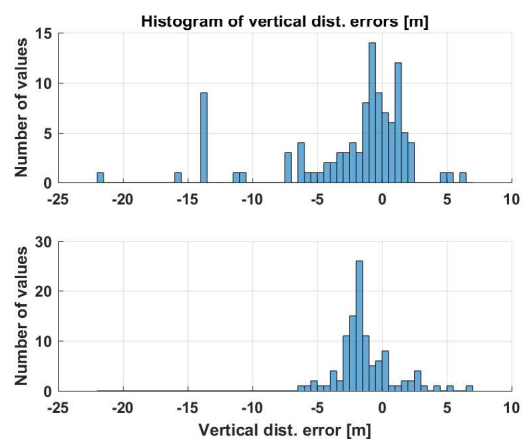

Fig. 20: Histogram of vertical distance estimation errors at decision for tower (upper for $15 \mathrm{~m} / \mathrm{s}$ lower for $30 \mathrm{~m} / \mathrm{s} V_{o}$ )

\section{CONCLUSION}

This paper made the first step towards the extension of the author's previous S\&A method (considering aircraft intruders) into the direction of application for ground obstacles. The previously derived formulae are extended to steady ground obstacles. Then a software-in-the-loop (SIL) MonteCarlo test campaign is done considering a car and a tower obstacle to show the capabilities of the developed method. The time to closest point of approach estimation results are really good the closest point of approach is usually underestimated but by selecting a proper threshold it can be well used to avoid dangerous (close) obstacles. The size, side, forward and vertical distances of the obstacles can be also estimated but their precision only make it possible to use them as references for the order of magnitude of these values. Future plans are to develop a proper avoidance strategy considering also the precision of the estimated parameters. After SIL testing real flight tests with a small UAV and artifical ground obstacles is planned to be done.

\section{REFERENCES}

[1] EU, "Roadmap for the integration of civil Remotely-Piloted Aircraft Systems into the European Aviation System," European RPAS Steering Group, Tech. Rep., 2013.

[2] Y. Lyu, Q. Pan, C. Zhao, Y. Zhang, and J. Hu, "Feature article: Visionbased UAV collision avoidance with 2D dynamic safety envelope," IEEE Aerospace and Electronic Systems Magazine, vol. 31, no. 7, pp. 16-26, July 2016.

[3] L. Mejias, A. McFadyen, and J. J. Ford, "Feature article: Sense and avoid technology developments at Queensland University of Technology," IEEE Aerospace and Electronic Systems Magazine, vol. 31, no. 7, pp. 28-37, July 2016.

[4] A. Nussberger, H. Grabner, and L. V. Gool, "Feature article: Robust Aerial Object Tracking from an Airborne platform," IEEE Aerospace and Electronic Systems Magazine, vol. 31, no. 7, pp. 38-46, July 2016.

[5] VISION project (Validation of Integrated Safetyenhanced Intelligent flight cONtrol). [Online]. Available: http://w3.onera.fr/h2020_vision/node/1

[6] S. Badal, S. Ravela, B. Draper, and A. Hanson, "A Practical Obstacle Detection and Avoidance System," in IEEE WACV, Sarasota, FL, 1994.

[7] R. Kikutis, J. Stankunas, D. Rudinskas, and T. Masiulionis, "Adaptation of Dubins Paths for UAV Ground Obstacle Avoidance When Using a Low Cost On-Board GNSS Sensor," Sensors, vol. 17, no. 10, pp. 1-23, 2017.

[8] O. Esrafilian and H. D. Taghirad, "Autonomous flight and obstacle avoidance of a quadrotor by monocular slam," in 2016 4th International Conference on Robotics and Mechatronics (ICROM), Oct 2016, pp. $240-245$.

[9] J. B. Saunders, O. Call, A. Curtis, A. W. Beard, and T. W. Mclain, "Static and dynamic obstacle avoidance in miniature air vehicles, in aiaa infotech at aerospace, 2005, paper no," in in Proceedings of the Infotech@Aerospace Conference, 2005, pp. 2005-6950.

[10] J. Saunders, R. Beard, and J. Byrne, "Vision-based reactive multiple obstacle avoidance for micro air vehicles," in 2009 American Control Conference, June 2009, pp. 5253-5258.

[11] P. Bauer, A. Hiba, B. Vanek, A. Zarandy, and J. Bokor, "Monocular Image-based Time to Collision and Closest Point of Approach Estimation," in In proceedings of 24th Mediterranean Conference on Control and Automation (MED'16), Athens, Greece, 2016.

[12] P. Bauer, A. Hiba, and J. Bokor, "Monocular Image-based Intruder Direction Estimation at Closest Point of Approach," in in Proc. of the International Conference on Unmanned Aircraft Systems (ICUAS) 2017. Miami, FL, USA: ICUAS Association, June 2017, pp. 11081117.

[13] P. Bauer and A. Hiba, "Vision Only Collision Detection with Omnidirectional Multi-Camera System," in in Proc. of the 20th World Congress of the International Federation of Automatic Control. Toulouse, France: IFAC, July 2017, pp. 15 780-15 785. 